RolX: Role Extraction and Mining in Large Networks

K. Henderson, B. Gallagher, T. Eliassi-Rad, H. Tong, L. Akoglu, D. Koutra, L. Li, S. Basu, C. Faloutsos

September 13, 2011 
This document was prepared as an account of work sponsored by an agency of the United States government. Neither the United States government nor Lawrence Livermore National Security, LLC, nor any of their employees makes any warranty, expressed or implied, or assumes any legal liability or responsibility for the accuracy, completeness, or usefulness of any information, apparatus, product, or process disclosed, or represents that its use would not infringe privately owned rights. Reference herein to any specific commercial product, process, or service by trade name, trademark, manufacturer, or otherwise does not necessarily constitute or imply its endorsement, recommendation, or favoring by the United States government or Lawrence Livermore National Security, LLC. The views and opinions of authors expressed herein do not necessarily state or reflect those of the United States government or Lawrence Livermore National Security, LLC, and shall not be used for advertising or product endorsement purposes.

This work performed under the auspices of the U.S. Department of Energy by Lawrence Livermore National Laboratory under Contract DE-AC52-07NA27344. 


\section{RolX: Role Extraction and Mining in Large Networks}

\author{
Keith Henderson \& Brian Gallagher \\ Lawrence Livermore Nat'l Lab \\ $\{$ keith,bgallagher\}llnl.gov \\ Leman Akoglu \& Danai Koutra \& Lei Li \\ Carnegie Mellon University \\ \{lakoglu,danai,leili\}@cs.cmu.edu
}

\author{
Tina Eliassi-Rad \\ Rutgers University \\ eliassi@cs.rutgers.edu \\ Sugato Basu \\ Google Research \\ sugato@google.com
}

\author{
Hanghang Tong \\ IBM Watson \\ htong@us.ibm.com
}

\author{
Christos Faloutsos \\ Carnegie Mellon University \\ christos@cs.cmu.edu
}

\begin{abstract}
Given a graph, how can we automatically discover roles for nodes? Roles could be, eg., 'bridges', or 'peripherynodes', etc. Roles are compact summaries of a node's behavior that generalize across networks. They enable numerous novel and useful network mining tasks, such as sense-making, searching for similar nodes, and node classification. We propose RolX (Role eXtraction), a scalable (linear in the number of edges), unsupervised learning approach for automatically extracting roles from general network data. We demonstrate the effectiveness of RolX on several network mining tasks, from exploratory data analysis to network transfer learning. Moreover, we compare network role discovery with network community discovery. We highlight fundamental differences between the two (e.g., roles generalize across disconnected networks, communities do not).
\end{abstract}

Keywords-Graph mining, role discovery

\section{INTRODUCTION}

Roles are compact summaries of behavior that generalize across networks. Examples of roles are active nodes, isolated nodes, broker nodes, peripheral nodes, etc. Given a graph, can we find the roles that nodes play in a graph - i.e., can we discover the behavior/function of a node? This problem, a.k.a. role discovery, is important because roles form a basis for a number of novel and interesting network data analysis tasks such as sense-making, neighborhood affinity of a node, and graph visualization. In addition, roles can be used to support other common graph mining tasks, such as searching for similar nodes and node classification. For instance, given two IP communication graphs from enterprise networks, we can use the extracted roles in one graph to build a relational classifier that can be used for a classification task in the other graph, effectively performing across-network classification or transfer learning on graphs.

We propose RolX (Role eXtraction), an unsupervised learning approach for automatically extracting roles from general network data sets. Our approach combines feature selection, feature grouping (through matrix factorization), and model selection in a novel way; the result is a unified method that is scalable (linear in the number of edges), completely automated, and effective. In addition, RolX is the first method to introduce a stopping criterion for non- negative matrix factorization, by carefully using Minimum Description Length (MDL).

Through extensive experiments, we demonstrate that the derived roles are effective in both exploratory data analysis tasks (such as node-similarity, neighborhood-affinity, and sense-making) and prediction tasks such as across-network transfer learning. In the latter setting, we use the ability of roles to generalize behavior across networks as a way to perform network learning without relying on homophily or on the availability of class labels in the target graph.

The contributions of our work are as follows:

- Effectiveness: RolX captures roles that agree with intuition and that are generalizable and useful in exploratory data analysis and prediction tasks. Moreover, it helps with understanding the graph ('sense-making'), by summarizing how nodes of different 'role' connect to each other.

- Scalability: RolX has runtime that is linear on the number of edges.

We want to emphasize that role discovery is fundamentally different from community detection: the former groups nodes of similar behavior; the latter groups nodes that are well connected to each other. We elaborate further, next.

\section{A. Role Discovery vs. Community Discovery}

Both role discovery and community discovery fall under the general task of graph clustering. However, they address very different tasks. Typically, community discovery approaches optimize for groups of nodes that have high intralink density and low inter-link density. Such communities do not generalize across disjoint networks. In other words, they cannot be used for prediction tasks across different networks. Role discovery, on the other hand, finds sets of roles denoting behaviors that nodes play in a graph. These roles generalize across various disconnected networks and (as we will show in Section III) can be used in exploratory data analysis such as sense-making and node-similarity across disconnected networks. Intuitively, roles generalize across networks because the set of roles that nodes play is often the same across networks. That is, networks often contain 
nodes that are for example 'centers of stars,' 'satellites of stars,' 'bridges/brokers,' etc.

Figure 1 depicts the difference between role discovery and community discovery for the largest connected component (LCC) of a weighted co-authorship network [1]. RolX discovers 4 roles vs. the 22 communities that the popular Fast Modularity [2] community discovery algorithm finds. The node colors for RolX correspond to the node's primary role, ${ }^{1}$ and for Fast Modularity correspond to the node's community. The four discovered roles represent these behaviors: bridge nodes (blue diamonds) representing central and prolific authors, main-stream nodes (green squares) representing neighborhoods of bridge nodes, "pathy" nodes (red triangles) representing peripheral authors, and "tight knit" nodes (black circles) representing authors with many coauthors and homophilic neighborhoods.
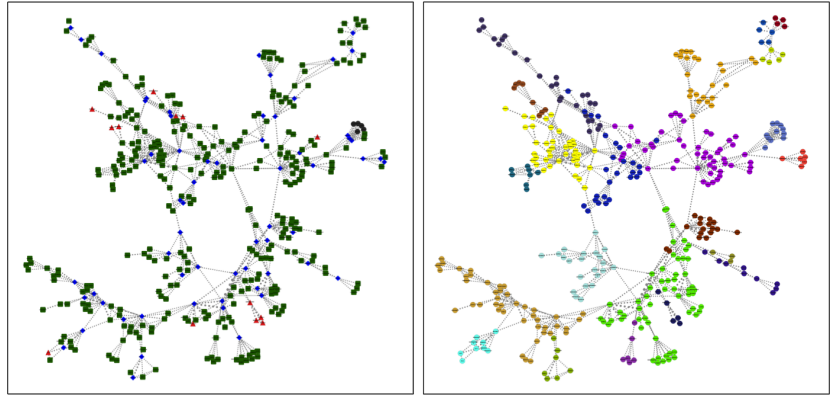

Figure 1. Fundamental differences between role discovery vs. community discovery. Left: the 4 roles that RolX discovers on the largest connected component (LCC) of the Network Science Co-authorship Graph: 'bridge' nodes (as blue diamonds), 'main-stream' nodes (green squares) etc - see text. Right: the 22 communities that Fast Modularity [2] finds on the same co-authorship graph. Roles capture node-level behaviors and generalize across networks whilst communities cannot.

The rest of the paper is organized into the following sections: proposed method, qualitative experiments on role effectiveness, quantitative experiments on role effectiveness, related work, and conclusions.

\section{Proposed Method}

Given a network, the goal of $\operatorname{RolX}$ is to automatically discover a set of underlying (latent) roles, which summarize the behavior of nodes in the network. RolX consists of three components: feature extraction, feature grouping, and model selection.

\section{A. Feature Extraction}

The first step is to describe each node as a feature vector. A feature could be, eg., the number of neighbors; another feature could be, eg., the number of triangles our node participates in. RolX can use any set of features we deem important. Among the numerous choices for feature

\footnotetext{
${ }^{1}$ RolX is a mixed-membership approach, which assigns each node a distribution over the set of discovered roles.
}

extraction functions, we choose the ReFex feature discovery algorithm [3], since it has shown good performance. In short, for a given node $n$, it extracts local and egonet features based on counts (weighted and unweighted) of links adjacent to $n$ and within and adjacent to the egonet of $n$. It also aggregates egonet-based features in a recursive fashion until no informative feature can be added.

\section{B. Feature grouping}

After feature extraction, we have $n$ vectors of $f$ numerical entries each. How should we create groups of nodes with similar behavior/features? How can we make it fully automatic, requiring no input from the user?

We propose to use soft clustering, and specifically, an automatic version of matrix factorization.

Given a node-feature matrix $V_{n \times f}$, the next step of the $\operatorname{RolX}$ algorithm is to generate a rank $r$ approximation $G F \approx V$ where each row of $G_{n \times r}$ represents a node's membership in each role and each column of $F_{r \times f}$ specifies how membership in a specific role contributes to estimated feature values. There are many methods to generate such an approximation (e.g., SVD, spectral decomposition) and RolX is not tied to any particular approach. For this study, we chose Non-negative Matrix Factorization (NMF) because it is computationally efficient and non-negative factors simplify the interpretation of roles and memberships.

Formally, NMF seeks two non-negative low rank matrices $G$ and $F$ to satisfy: $\operatorname{argmin}_{G, F}\|V-G F\|_{\text {fro }}$, s.t. $G \geq$ $0, F \geq 0$. The non-negativity constraint generally leads to a sparse, part-based representation of the original data set, which is often semantically more meaningful than other factorization methods. While it is difficult to find the optimal solution of NMF because of the non-convexity of the objective function, several efficient approximation algorithms exist (e.g., multiplicative update [4], projective gradient decent [5]). Here we use multiplicative update because of its simplicity. It is worth pointing out there exist several variants of NMF (e.g., using Bregman divergence to measure the approximation accuracy [6], imposing sparseness constraint on $F$ and/or $G$ by incorporating some regularization terms in the objective function [7]). All such variants can be naturally plugged into our proposed framework.

One practical issue with NMF is that the model size (i.e., the number of roles) must be pre-specified. In general, it is unrealistic to expect a practitioner to manually select an appropriate value for this parameter. Therefore, we explore several approaches for automatic model selection. To our knowledge, we are the first to propose a model selection criterion for NMF.

\section{Automating the method - Model Selection}

Our goal is to have no input from the user, and instead, have $\operatorname{RolX}$ automatically decide the number of roles $r$. We briefly describe a few such criteria. 
1) Predictive Performance: If external node attributes are available, one can choose the $r$ that performs best at predicting these attributes (using an appropriate measure of predictive quality). See Figure 8 for an example of how classification accuracy in an IP network varies with $r$.

2) Akaike Information Criterion: When no attributes are available or predictive performance is not an acceptable criterion, there are a number of criteria that can be applied to select $r$. A popular measure is the information criterion proposed by Akaike (AIC) [8], which penalizes models for having a large number $k$ of parameters, and for low maximized likelihood $L$. The AIC is defined as $A I C=2 k-2 \ln (L)$ For a given $G$ and $F$ computed using NMF with rank $r$, we have:

$$
\begin{gathered}
k=r(n+f) \\
\ln (L)=-\frac{1}{2 \sigma^{2}}\|V-G F\|_{F}^{2}
\end{gathered}
$$

Here $\|\cdot\|_{F}$ is the Frobenius norm and $\sigma^{2}$ is the variance of the values in $V$. This expression for likelihood assumes that the noise in the data is Gaussian.

3) Compression: Since roles summarize behavior, they can be used to compress the feature matrix $V$. We propose to use the Minimum Description Length criterion [9], to select the model size $r$ that results in best compression. For a given model, we can compute the resulting description length in two parts: (1) the number of bits required to describe the model itself, and (2) the cost of describing the reconstruction errors in $V-G F$ (to achieve lossless compression). The selected model is the one that minimizes the description length $\mathcal{L}$, which is the sum of model description cost $\mathcal{M}$ and the coding cost (or equivalently, the cost of correcting the errors of our model) $\mathcal{E}$

$$
\mathcal{L}=\mathcal{M}+\mathcal{E}
$$

Assuming $G$ and $F$ are not sparse, the cost of describing the model using $b$ bits per value is

$$
\mathcal{M}=\operatorname{br}(n+f)
$$

Because the model can contain high-precision floating point values, we combine Lloyd-Max quantization [10], [11] with Huffman codes [12] to increase compression. Note that with Huffman codes, the cost of encoding the model changes to $\bar{b} r(n+f)$, where $\bar{b}$ is the mean number of bits required per value. As a default, we choose $\log _{2}(n)$ quantization bins. Preliminary analysis suggests that the selected model is not sensitive to this parameter for a large range of values.

We explored other options for compressing $G$ and $F$. Sparse NMF [7] generates a model with a smaller number of nonzeros, so that the model can be described sparsely. However, we found that in most cases the number of zeros was not high enough to offset the additional cost of specifying positions in $G$ and $F$. We also used rounding instead of quantization to compress the floating point values in the model, but found that these models have higher error than quantized models for the same model size in bits.

Again assuming Gaussian noise, we can approximate the cost of encoding errors by computing the log likelihood of $V$ in the model in bits. Using $\ln (L)$ as defined in (2), this can be expressed as

$$
\mathcal{E}=\log _{2}(e) * \ln (L)
$$

4) Remarks: The goal of MDL is to balance model complexity with model errors, both in terms of selecting $r$ and in terms of the representation chosen for values in $G$ and $F$. We find that quantization and Huffman coding typically outperform other representations, but MDL is compatible with a wide variety of model descriptions. For this reason, we prefer MDL to the Akaike criterion, although in practice they often agree or nearly agree on model size. Throughout this paper, we refer the to RolX algorithm that uses MDL as $\operatorname{Rol} X-M$ and the RolX algorithm that uses AIC as $\operatorname{Rol} X-A$. When no qualifier is attached (i.e., just $\operatorname{Rol} X$ ), assume the default of MDL.

\section{Computational Complexity}

Let $n$ be the number of nodes, $m$ be number of edges, $f=$ number of features, and $r=$ number of roles.

Lemma 1: The running time complexity of RolX is linear on the number of edges, and specifically

$$
O(m f+n f r)
$$

Proof: We give the complexity of each of the three steps of RolX.

Feature Extraction. This is $O(f(m+n f))$ [3].

Model Selection. The AIC and MDL error computations each take $O(n r f)$ to multiply an $n \times r$ matrix by an $r \times f$ matrix. MDL has an additional quantization complexity of $O(n f I \log (K))$, where $K$ is the number of quantization bins and $I$ is the number of iterations that we run the quantizer. Default for $K$ is $\log (n)$, so this becomes $O(n f I \log (\log (n)))$. Notice that the term of $O(\log (\log (n)))$ is a very small number. For example, for a graph with 1 billion nodes, this term is just about 3.1 . So, the complexity for the quantization is roughly $O(n f I)$. There is also a term for the Huffman coding which is $O(n f+K \log (K))$. The $O(K \log (K))$ term is to build the tree that holds the codes. But this number is very small and therefore can be neglected.

Feature Grouping. We use the multiplicative update method of NMF, which has worst case complexity $O(n f r+$ $\left.n r^{2}+f r^{2}\right)=O(n f r)$.

\section{QuAlitative EXPERIMENTS ON ROLE EFFECTIVENESS}

In this section, we present qualitative results on experiments of role effectiveness for clustering. 


\section{A. Role Discovery}

1) Network Science: Our first data set is the weighted Network Science Co-authorship Graph with 1589 authors (from the network science community) and 2743 weighted edges [1]. Figure 2 shows (a) the role-colored graph (where each node is colored by the primary role that RolX finds) and (b) the role affinity heat map. RolX finds four roles. The black circle and red triangle roles are very homophilous i.e., a black circle is mostly a neighbor of another black circle. The blue diamond role is the least homophilous. In fact, most blue diamonds are brokers/bridges and are neighbors of green rectangles.

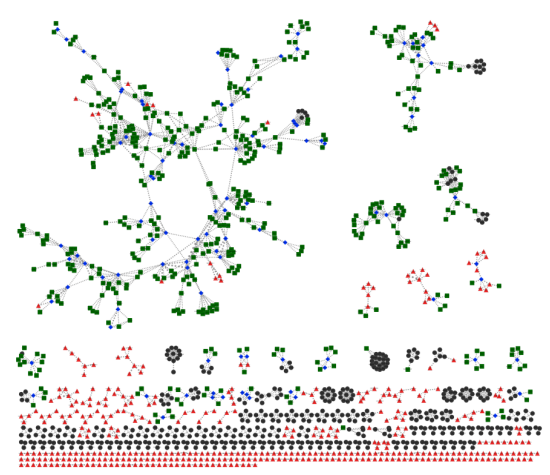

(a) Role-colored Visualization of the Network

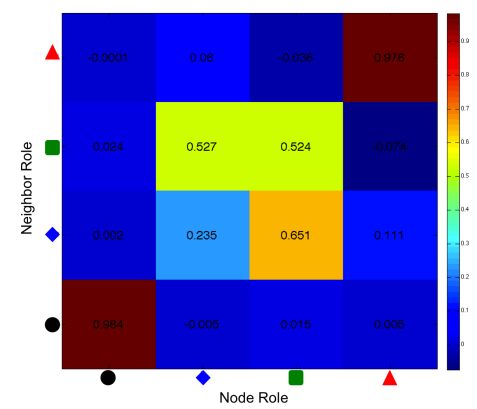

(b) Role Affinity Heat Map

Figure 2. RolX effectively discovers roles in the Network Science Coauthorship Graph. (a) Author network RolX discovered four roles, like the heterophilous bridges (blue diamond), as well as the homophilous "pathy" nodes (red triangle) (b) Affinity matrix (red is high score, blue is low) strong homophily for roles \#1 and \#4.

2) Political books: We gave the 2000 Amazon Political Books Co-purchasing Network ${ }^{2}$ to RolX-A. This graph has 105 nodes (representing books) and 441 edges (representing frequent co-purchasing of the books by the same buyers). RolX-A is able to effectively capture the purchasing behavior of customers and find the noise in the human-provided labels $^{3}$ (see Figure 3). The layout in Figure 3 groups the

\footnotetext{
${ }^{2}$ Valdis Krebs, http://www.orgnet.com/.

${ }^{3} \mathrm{An}$ example of the noise includes a label of conservative for "Bush at War," written by Bob Woodward who is generally considered a neutral to liberal-leaning author.
}

nodes by their noisy human-provided labels. The nodecolor corresponds to RolX-A's discovered roles. The red and blue roles, respectively, correspond to "main-stream" conservative and liberal books. The black role corresponds to books at the extreme fringe. As expected both the conservative and the liberal books have nodes that have the black role. Examples include "Off with Their Heads" and "Bush Country" on the extreme conservative side and "The Lies of George W. Bush" and "Worse Than Watergate" on the extreme liberal side. The green role corresponds to bridges (or brokers).

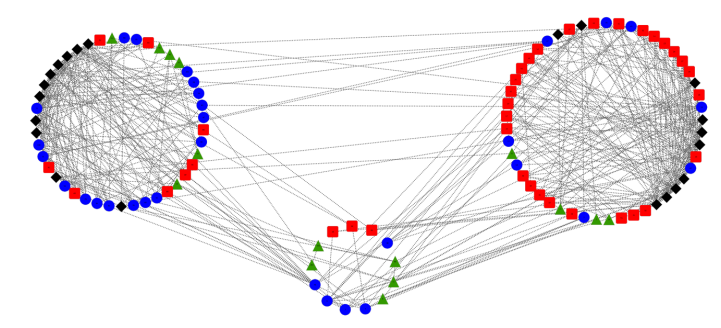

Figure 3. RolX effectively discovers roles in the 2000 Amazon Political Books Co-purchasing Network. RolX-A finds four roles: main-stream conservative (red), main-stream liberal (blue), extreme fringe (black), and bridge (green). The layout corresponds to the human-provided labels of conservative (the circle on the right), liberal (the circle on the left), and neutral (the circle in the middle).

\section{B. Node Similarity}

RolX's roles allow us to find similar nodes by comparing their role distributions. Figure 4 depicts node similarity for three (target) authors for the Network Science Co-authorship Graph: Mark Newman, F. Robert, and J. Rinzel. The primary roles for these three authors are different. Mark Newman's primary role is a broker (a prolific author); F. Robert's primary role places him in a tight-knit group (an author with homophilous neighborhood), and J. Rinzel's primary role places him in the periphery (an author with homophilous but "pathy" neighborhood). In each node-similarity picture, the target author is colored in yellow. The more similar nodes are red and less similar nodes are blue. Due to the generality of roles, RolX is able to find similar nodes across the entire graph even though it has many disconnected components.

Figure 5 depicts node similarity for two conservative books in the 2000 Amazon Political Books Co-purchasing Network. One with the fringe role (titled "Off with Their Heads") and another with the bridge role (titled "We Will Prevail"). In each picture, the target node is colored yellow. The more similar nodes are red and less similar nodes are blue. For the conservative fringe "Off with Their Heads" book, RolX-A finds fringe conservative and liberal books that are similar to this book but no neutral books. The four most similar books are fringe conservative books ("A National Party No More," "Losing Bin Laden," "Slander," 


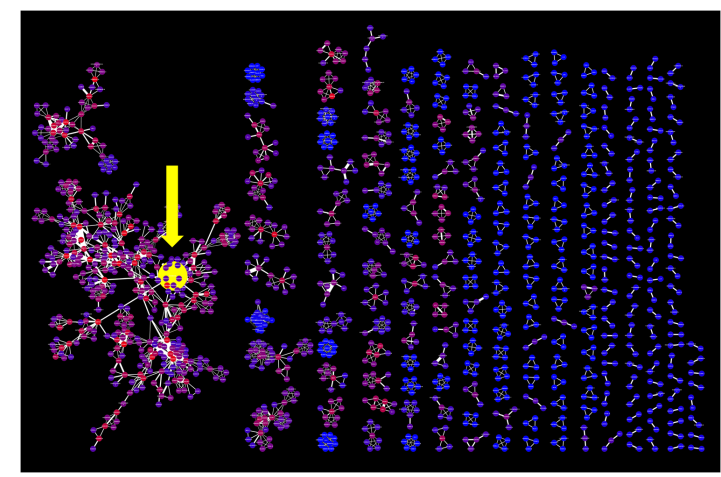

(a) Node similarity for Mark Newman

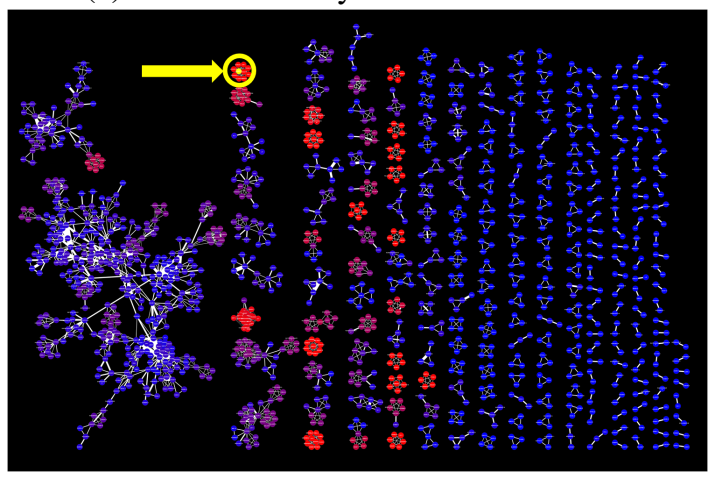

(b) Node similarity for F. Robert

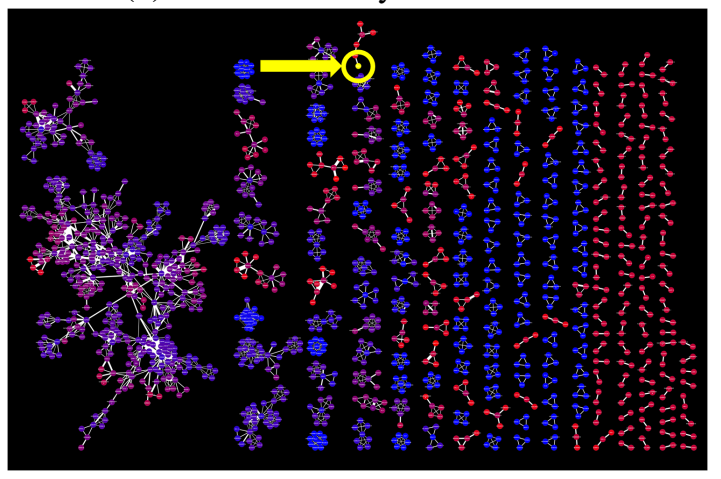

(c) Node similarity for J. Rinzel

Figure 4. RolX produces meaningful node-similarity across disconnected components of a graph. (a) Node similarity for Mark Newman (who is a broker). (b) Node similarity for F. Robert (who is from a tight-knit and homophilous neighborhood). (c) Node similarity for J. Rinzel (who is from a "pathy" but homophilous neighborhood). In each node-similarity picture, the target author is colored in yellow. The more similar nodes are red and less similar nodes are blue.

and "The Man Who Warned America"), followed by several fringe liberal books ("America Unbound," "The Choice," and "Bushwhacked"). For the conservative bridge "We Will Prevail" book, RolX-A finds other bridge books (mostly books labeled as neutral) and none of the fringe conservative or liberal books.

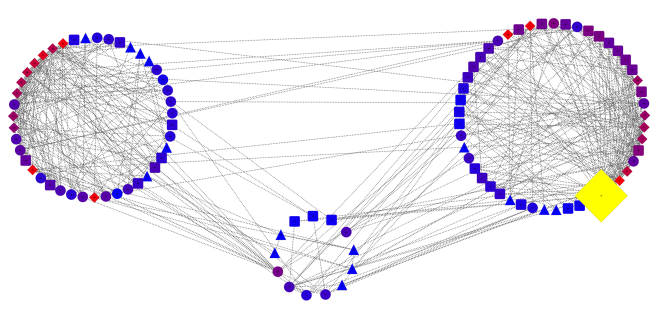

(a) Node similarity for the conservative fringe book "Off with Their Heads"

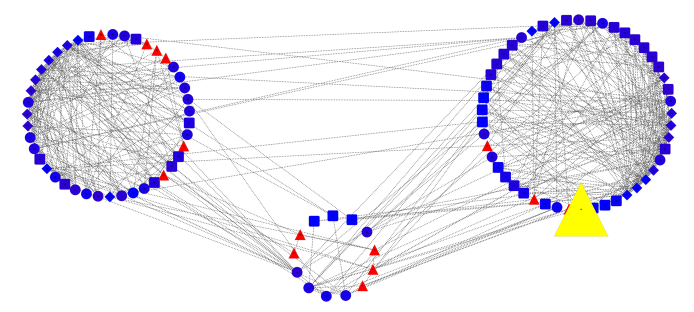

(b) Node similarity for the conservative bridge book "We Will Prevail"

Figure 5. RolX identifies similar nodes in the 2000 Amazon Political Books Co-purchasing Network. RolX-A finds other similar fringe books for a fringe book and other similar bridge books for a bridge book. It is able to clearly distinguish between these two roles to effectively answer a nodesimilarity query. The layout corresponds to the human-provided labels of conservative (the circle on the right), liberal (the circle on the left), and neutral (the circle in the middle). The target node is colored in yellow. The more similar nodes are red and less similar nodes are blue.

\section{Sense Making}

To make "sense" of roles, we introduce two methods. One based on node measurements (NodeSense) and another based on neighbor measurements (NeighborSense).

NodeSense takes as input RolX's node-by-role matrix, $G$, and a matrix of node measurements, $M$. For our experiments, we used the following node measurements: eccentricity (i.e., the longest geodesic from a node), number of biconnected components to which a node belongs ( $\# B C C$ ), a node's degree, its weighted degree, its clustering coefficient $(C C)$, and its PageRank. NodeSense then computes a matrix $E$ using NMF such that $G \cdot E \approx M$. The matrix $E$ represents the role contribution to node measurements. A default $E^{\prime}$ is also computed by using $G^{\prime}=$ ones $(n, 1)$, where the $n$ nodes belong to one role. Then, for each role $r$ and for each measurement $s$, NodeSense computes $\frac{E(r, s)}{E^{\prime}(r, s)}$. This ratio gives us the role-contribution to node-measurements compared to the default contribution.

NeighborSense is similar to NodeSense-except instead of the matrix $M$, we use a neighbor matrix $N$, where the rows represent nodes and columns represent roles and $N(i, j)$ is the fraction of node $i$ 's neighborhood that is in role $j$. Then NeighborSense computes a matrix $Q$ using NMF such that $G \cdot Q \approx N$. The matrix $Q$ represents the role affinities. A default $Q^{\prime}$ is also computed using $G^{\prime}=$ ones $(n, 1)$, where 
the $n$ nodes belong to one role. Then, for each pair of roles $r_{1}$ and $r_{2}$, NeighborSense computes $\frac{Q\left(r_{1}, r_{2}\right)}{Q^{\prime}\left(r_{1}, r_{2}\right)}$. This ratio gives us the role-affinities compared to the default affinities.

Figure 6 depicts the results of NodeSense and NeighborSense on the Network Science Co-Authorship Graph (see Figure 2a for its role-colored graph). If you recall, RolX found four roles on this graph:

The black circle and red triangle roles are very homophilous - i.e., a black circle is mostly a neighbor of another black circle. The blue diamond role is the least homophilous. In fact, most blue diamonds are brokers/bridges and are neighbors of green rectangles. Based on further analysis of the network and its roles, we discovered that the roles of RolX correspond to the following: (Disclaimer: The relative position in the graph does not reflect the total magnitude of contributions of the individual researchers. It is just a snapshot of networks-science-related data, and specifically in 2006.)

- black circle: tightly knit, nodes that participate in tightly-coupled groups. Examples are Andrei Broder and Christos Faloutsos.

- blue diamond: bridge nodes, that connect groups of (typically, 'main-stream') nodes. Examples of bridges are Albert-Laszlo Barabasi and Mark Newman.

- green rectangle: main-stream, the vast majority of nodes - neither on a clique, nor a chain. Examples are John Hopcroft and Jon Kleinberg.

- red triangle: pathy, nodes that belong to elongated clusters. For example, Lada Adamic and Bernardo Huberman.

In section IV-D, we present more sense-making results on a different data set (network trace data) as part of making sense of classification results.

\section{QuANTITATIVE EXPERIMENTS ON ROLE EFFECTIVENESS}

In this section, we present experiments on role effectiveness for the across-network classification task (i.e. network transfer learning) and report results on two real-world data sets: IP communication networks and bluetooth proximity networks.

\section{A. Data}

IP data: IP-A and IP-B are real network-trace data sets collected roughly one year apart on separate enterprise networks. The nodes are IP addresses and the links are communications between the IPs. The IP-A trace begins at midnight on day 1 and continues up to $12 \mathrm{pm}$ on day 5 . The IP-B trace begins at midnight on day 1 and continues up to $\approx 5 \mathrm{pm}$ on day 6 . For days $1-4$ of the IP-A dataset (IP-A1 to IP-A4), we extract flows in the period from $12 \mathrm{pm}-1 \mathrm{pm}$. We exclude day 5 because the trace ended at $12 \mathrm{pm}$. For IP-B, we extract flows from $12 \mathrm{pm}-1 \mathrm{pm}$ for day 3 only. We then label all flows using a payload signature-based classification tool.

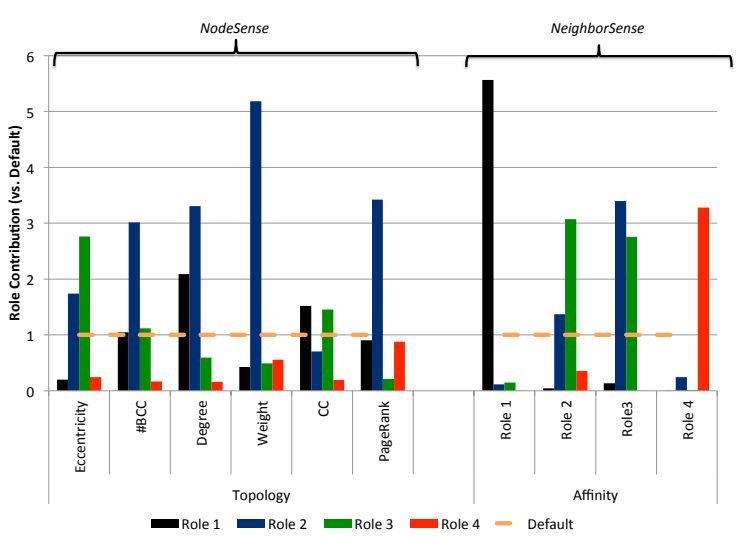

Figure 6. NodeSense and NeighborSense explain roles in the network science co-authorship graph. Role 1 depicts authors who have many coauthors and have homophilic neighborhoods (represented as black circles in Figure 2a). Role 2 represent authors who are central and prolific with high total weight (represented as blue diamonds in Figure 2a). Role 3 are peripheral authors (represented as green squares in Figure 2a). Role 4 are isolated authors with homophilic neighborhoods (represented as red triangles in Figure 2a).

\begin{tabular}{c|ccccc} 
& IP-A1 & IP-A2 & IP-A3 & IP-A4 & IP-B \\
\hline \# Nodes & 81,450 & 57,415 & 154,103 & 206,704 & 181,267 \\
(\# labeled) & 29,868 & 16,112 & 30,955 & 67,944 & 27,649 \\
\# Links & 968,138 & 432,797 & $1,266,341$ & $1,756,082$ & $1,945,215$ \\
(\# unique) & 206,112 & 137822 & 358,851 & 465,869 & 397,925 \\
\% Web & $32 \%$ & $38 \%$ & $38 \%$ & $18 \%$ & $42 \%$ \\
\% DNS & $36 \%$ & $49 \%$ & $39 \%$ & $20 \%$ & $42 \%$ \\
\% P2P & $32 \%$ & $12 \%$ & $23 \%$ & $62 \%$ & $16 \%$
\end{tabular}

Table I

EXTRACTED REAL-WORLD NETWORK TRACE DATA

Once network flows are labeled, we transfer labels to hosts by selecting the most frequent class-labels from among the host's flows. The payload classifier can distinguish between over 15 classes of traffic (e.g., Web, DNS, SMTP, P2P). However, since we found that 3 classes (namely, Web, DNS, and P2P) made up the dominant traffic type for over $90 \%$ of the labeled hosts, we remove all other labels and focus on the 3-class classification problem. Table I summarizes the data that we extracted.

Reality Mining Device data: This dataset is constructed based on the data provided by the Reality Mining project [13]. That study was conducted in July 2004-June 2005 at the MIT Media Laboratory, with the participation of 94 human subjects using mobile phones pre-installed with several pieces of software, which recorded and sent to the research center various data including information about Bluetooth devices in proximity of approximately 5 meters. Subjects were tracked over 12 months and included students and faculty from the MIT Media Lab and the Sloan Business 


\begin{tabular}{|c|c|c|c|c|c|}
\hline & \multicolumn{4}{|c|}{ Test Graph } \\
\hline & & IP-A2 & IP-A3 & IP-A4 & IP-B \\
\hline \multirow{5}{*}{ 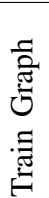 } & IP-A1 & $\checkmark$ & $\checkmark$ & $\checkmark$ & $\checkmark$ \\
\hline & IP-A2 & & $\checkmark$ & $\checkmark$ & $\checkmark$ \\
\hline & IP-A3 & & & $\checkmark$ & $\checkmark$ \\
\hline & IP-A4 & & & & $\checkmark$ \\
\hline & IP-A1 to IP-A4 & & & & $\checkmark$ \\
\hline & & Table & & & \\
\hline
\end{tabular}

ACROSS-NETWORK EXPERIMENTS PERFORMED ON THE NETWORK TRACE DATA

School. Within that period, about two million device scans were reported.

\section{B. Classifiers}

To test the predictive ability of the roles discovered by $\operatorname{RolX}$, we experiment with two variations of logistic regression. The first is a standard (non-regularized) logistic regression (LR). The second is the logForest (LF) model described by Gallagher et al. [14]. The logForest is a bagged model, composed of a set of logistic regression (LR) classifiers, where each is given a subset of $\log (f)+1$ of the $f$ total features. For our experiments, we use a logForest of 500 LR classifiers. The classifiers we compare are:

- RolX-LR: a logistic regression model, which uses RolX role memberships as features,

- RolX-LF: a $\log F$ rest model, which uses RolX role memberships as features,

- ReFeX: a logForest model, which uses the ReFeX regional features. Note that for ReFeX, logForest achieves vastly superior classification accuracy than logistic regression. Therefore, we omit the logistic regression based ReFeX model here.

Note that standard relational classifiers are not applicable for these transfer learning tasks since these methods rely on the availability of some known class labels in the test graph to seed the inference process. The test graphs here are completely unlabeled.

\section{Methodology}

For each experiment, the training graph has all known labels available. The test graph is completely unlabeled. Each classifier is evaluated on all known ground truth labels in the test graph. We use an identical set of roles for all data sets, which comes from running RolX on the IP-A1 data set. Table II summarizes the across-network experiments.

\section{Results}

IP data: Figure 7 demonstrates the performance of RolX and $\mathrm{ReFeX}$ on a series of across-network transfer learning tasks. We train on one network where all known labels are available, and test on a separate network that is completely unlabeled. These tasks are difficult, given the (sometime extreme) differences in class distributions between data sets

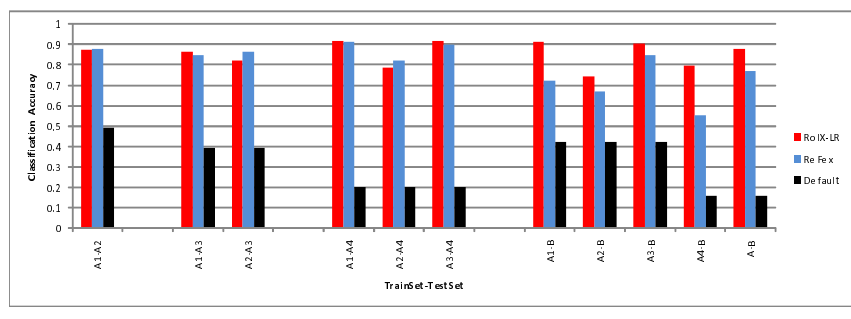

Figure 7. RolX provides better generalization performance between enterprise IP networks A and B (mean accuracy of $\operatorname{RolX}=85 \%, \mathrm{ReFeX}=71 \%$ acc., $\mathrm{p}=0.01$ ). Figure shows results for across-network transfer learning with RolX and ReFeX.

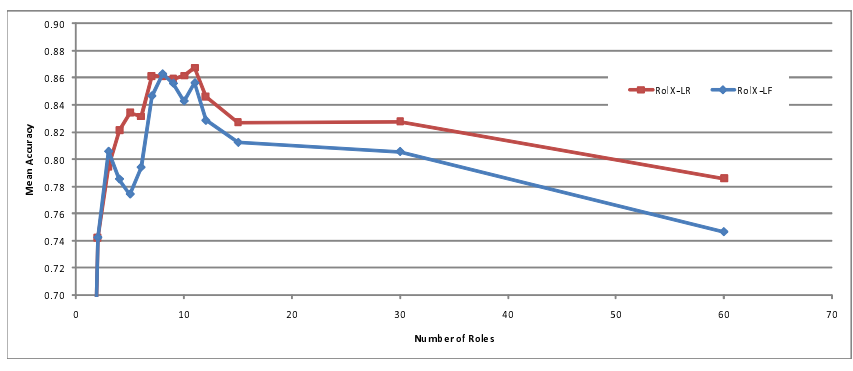

Figure 8. RolX-M and RolX-A agree on a high accuracy model size of 9 roles. Figure shows mean classification accuracy using RolX (over all 4 test sets) by model size.

(see Table I). The performance of the Default classifier is a good indicator of the difficulty of each task, since this model makes predictions based solely on the most frequent class from the training set.

ReFeX uses the full set of 373 regional features that it extracted from IP-A1. RolX summarizes these features into 9 roles. This model size was selected automatically by both MDL and AIC.

We see from Figure 7 that the roles produced by RolX effectively summarize the behavior of hosts in an IP network. Both RolX and ReFeX achieve equivalent performance transferring knowledge within the same enterprise network (mean accuracy of $\operatorname{RolX}=86 \%, \mathrm{ReFeX}=87 \%, \mathrm{p}=0.25$ ), but RolX is able to generalize more effectively from network A to network B ( $R o l X=85 \%, \operatorname{ReFeX}=71 \%$ acc., $\mathrm{p}=0.01)$.

Figure 8 demonstrates that both $\operatorname{RolX}-M$ and RolX-A are quite effective for our IP network classification task. Classification accuracy peaks in the 8-11 role range - both RolX-M and RolX-A select a high accuracy model size of 9 roles. In addition, Figure 9 shows that the criteria used by both $\operatorname{RolX}-M$ and $R o l X-A$ are correlated with classification accuracy - Pearson correlation for RolX-M is -0.80 and for RolX-A is -0.93 .

Figure 10 shows that IP traffic classes are well-separated in the RolX "role space", with as few as 3 roles (extracted from ReFeX's 373 original structural features). Note that we achieve even better separation with the automatically selected model size of 9 roles (see Figure 7), but we can 


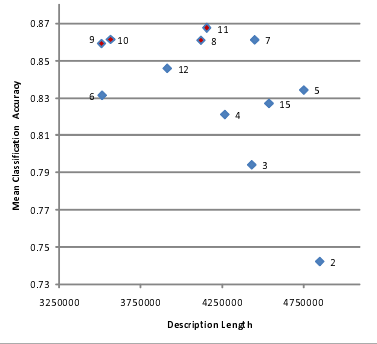

(a)

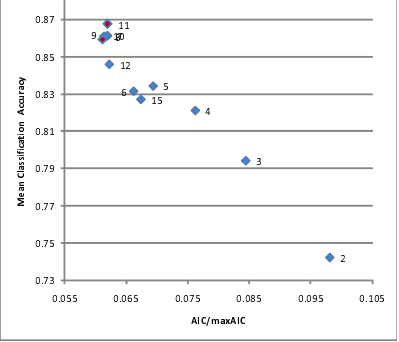

(b)
Figure 9. Our model selection works: Both (a) RolX-M and (b) RolX$A$ criteria give better results when they are minimized - giving higher mean classification accuracy (over all 4 test sets). Red markers indicate best performing model sizes ( $8-11$ roles).

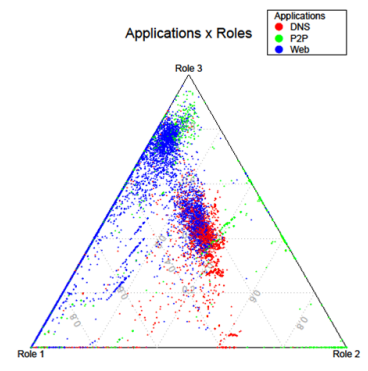

(a)

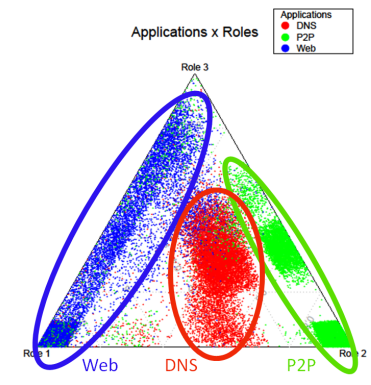

(b)
Figure 10. IP traffic classes are well-separated in the RolX "role space" with as few as 3 roles. (a) Ternary plot showing the degree of membership of each DNS, P2P, and Web host in each of Roles 1, 2, and 3. (b) Pseudodensity plot obtained by adding uniform noise to (a) to reveal overlapping points.

only clearly visualize up to 3 .

Figure 11 provides a "sense-making" explanation for the RolX results on the IP network data (when we tell it to find 3 roles as opposed to letting model selection pick the appropriate number of roles, which in this case was 9). Role 1 is Web clients. Role 2 is low-volume P2P users. Role 3 is a mixture of Web servers, DNS servers, and high-volume P2P nodes. This third Role is overloaded since the model size of 3 is not as predictive as larger model sizes (see Figure 8).

Figure 12 compares the performance of RolX-LR, which uses standard logistic regression, to RolX-LF, which uses the $\log$ Forest model. We see that $R o l X-L F$ is slightly, but significantly, better than RolX-LR when trained and tested on network A (mean diff $=0.02, \mathrm{p}=0.047$ ). However, RolX$L R$ outperforms RolX-LF when generalizing from network A to $\mathrm{B}$ (mean diff $=0.07, \mathrm{p}=0.025$ ). This result is expected since, the strength of $\operatorname{RolX}-L F$ is its ability to implicitly perform feature selection. Since we have so few features here, RolX-LF provides only a small benefit within network $A$ and leads to a less general model, which is less accurate than RolX-LR when applied to network B.

Reality Mining Device data: Using the Reality Mining

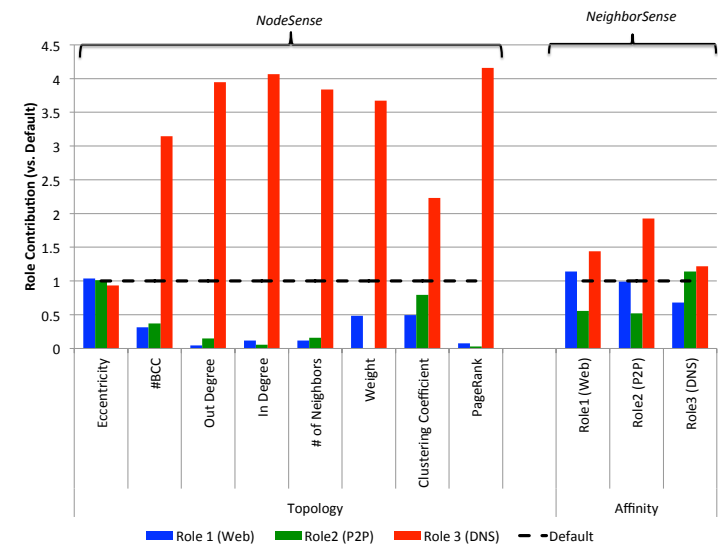

Figure 11. NodeSense and NeighborSense explain roles in IP networks. An examination of the contributions of different roles based on topology and affinity shows that Web servers are homophilous and have higher weighted degree; P2P clients have an affinity for DNS servers and have higher clustering coefficient; and DNS servers share properties of Web servers and $\mathrm{P} 2 \mathrm{P}$ clients.

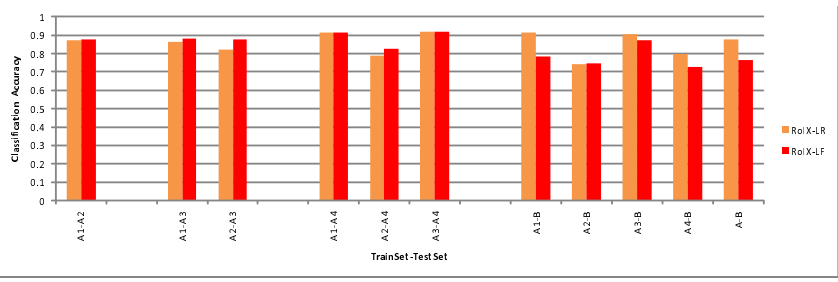

Figure 12. RolX-LR provides better generalization performance between enterprise IP networks A and B. Figure shows results for across-network transfer learning with $\operatorname{RolX}-L R$ and $\operatorname{RolX}-L F$.

Device dataset, we conducted two sets of transfer learning experiments. The first set of experiments involves a binary classification task where we try to predict whether a given subject is a business school student or not. The second set is similar, where we try to predict whether a subject is a graduate student in the Media Lab or not. As train and test sets, we used each pair of consecutive months in our dataset. In Figure 13, we show the accuracy of RolX. The Baseline is a classifier that learns to always predict the majority class of the training set on the test set. The time labels denote the month for the train data, and note that the month following that is used as the test data. We also use all the data in 2004 and 2005 as train and test data, respectively. Notice that RolX most of the time outperforms the baseline classifier with an average of $79 \%$ and $75 \%$ accuracy for the two experiment sets, respectively. We notice that RolX accuracy drops when September and May data is used as training, possibly because these months correspond to the start and end of the school semesters; the behavior of the subjects 


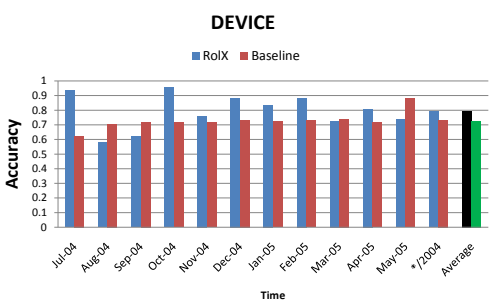

(a) Business Student vs. Rest

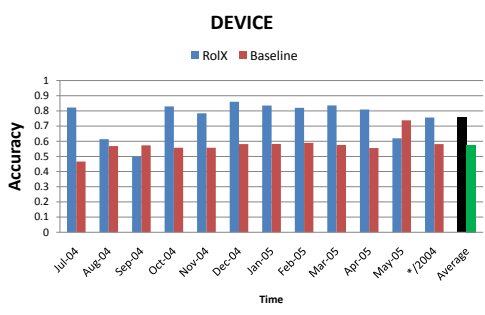

(b) Graduate Student vs. Rest

Figure 13. RolX (in blue) effectively generalizes behavior across time (higher is better). Figure shows results of across-network transfer learning on the Reality Mining Device dataset with RolX. Notice that RolX performs better at almost all times on two different learning tasks with an average accuracy of $79 \%$ and $75 \%$, respectively.

would be generally different than usual in these months, thus providing not as much predictive information as the other months would.

\section{RELATED WORK}

In this section, we discuss related research corresponding to different aspects of our work, which can be categorized into three parts: (1) graph features, (2) role discovery, and (3) transfer learning.

Graph Features. Features have been extracted from graphs for several data mining tasks. In [15], the authors propose extracting topological features for pairs of nodes for link prediction. In [16], the authors develop a multi-level framework to detect anomalies in time-varying graphs based on graph, sub-graph, and node level features. The algorithms rely on extracting graph-level (global) features and tracking these metrics over time. In [17], the authors propose to detect abnormal nodes from weighted graphs based on features and patterns from the egonet. There has also been work on using local and global structural features to improve the performance of network classifiers [18]. Some methods for feature extraction explicitly preserve the multi-cluster structure in the data [19]. Our work builds upon the approach of recursive feature extraction [3]. There also exists work to extract structural features (e.g., frequent sub-graphs) from the attributed graphs [20], [21].

Role discovery. We propose using recursive graph features for role discovery of nodes. The task of role discovery has been studied in different types of graphs, e.g., social networks [22]. Different approaches have been used for role discovery, including Bayesian frameworks using MCMC sampling algorithm for learning multiple roles of data points [23], semi-supervised semantic role labeling [24], etc. There is another related body of work in role mining, a nice overview of which is given by Molloy et al. [25]. Role mining is somewhat different from the role discovery problem we discuss in this paper - it addresses the access permissions for different users in different roles in an accesscontrolled system. However, the role mining algorithms use techniques similar to role discovery for inferring roles for nodes in a graph, e.g., hierarchical clustering.

In this paper we also use the inferred roles for improving classification. Previous approaches include using cluster structure for predicting class labels on graphs [26], and using cluster kernels for semi-supervised classification [27]. Our approach is scalable to large graphs due to the scalability of the underlying role discovery approach, and outperforms other state-of-the-art algorithms in classification tasks over graphs.

Transfer learning. Another aspect of our work is effective transfer learning in graphs. In the context of graph data, non-parametric models have been proposed for transfer learning for the task of collective link prediction [28]. The general framework of EigenTransfer constructs a graph to represent the source-target transfer learning task [29], while similarity matrix approximations of a hybrid graph have been explored for transfer learning [30]. Relevant supervision has also been transferred across domains to improve the performance of clustering algorithms [31]. It is worth pointing out that in all existing work, the features are given as the input and the goal is to leverage the given features to boost performance in the target domain. In this paper, we show that the discovered roles by our RolX can be used as a very powerful feature to improve the performance of such transfer learning.

\section{CONCLUSIONS}

We presented RolX, which matches the desirable properties we mention in the introduction:

- Effectiveness: RolX automatically extracts roles from general network data. The derived roles agree with intuition and perform well for graph mining tasks (e.g., classification, transfer learning)

- Scalability: RolX is linear on the number of edges.

Through extensive experimental validation on several real datasets, we showed that the extracted roles generalize across networks in numerous graph mining tasks, from exploratory data analysis to network transfer learning.

\section{ACKNOWLEGEMENTS}

This work performed under the auspices of the U.S. Department of Energy by Lawrence Livermore National 
Laboratory under Contract DE-AC52-07NA27344 (LLNLTR-498952).

\section{REFERENCES}

[1] M. E. J. Newman, "Finding community structure in networks using the eigenvectors of matrices," Phys. Rev. E., vol. 74, p. 036104, 2006.

[2] A. Clauset, M. Newman, and C. Moore., "Finding community structure in very large networks," Phys. Rev. E, vol. 70, p. 066111, 2004

[3] K. Henderson, B. Gallagher, L. Li, L. Akoglu, T. Eliassi-Rad, H. Tong, and C. Faloutsos, "It's who you know: Graph mining using recursive structural features," in KDD, 2011.

[4] D. D. Lee and H. S. Seung, "Learning the parts of objects by non-negative matrix factorization," Nature, vol. 401, no. 6755, pp. 788-791, 1999.

[5] C.-J. Lin, "Projected Gradient Methods for Nonnegative Matrix Factorization," Neural Computation, vol. 19, no. 10, pp. 2756-2779, 2007.

[6] I. S. Dhillon and S. Sra, "Generalized nonnegative matrix approximations with Bregman divergences," in NIPS, 2005, pp. 283-290.

[7] J. Eggert and E. Korner, "Sparse coding and nmf," in IJCNN, 2004, pp. 2529-2533.

[8] H. Akaike, "Information theory and an extension of the maximum likelihood principle," in Proc. of the 2nd Int'l Symp. on Information Theory, 1972, pp. 267-281.

[9] J. Rissanen, "Modeling by shortest data description," Automatica, vol. 14, pp. 465-471, 1978.

[10] J. Max, "Quantizing for minimum distortion," IRE Trans. on Information Theory, vol. IT-6, pp. 7-12, 1960.

[11] S. P. Lloyd, "Least squares quantization in PCM," IEEE Trans. on Information Theory, vol. IT-28, no. 2, pp. 129-137, 1982.

[12] D. Huffman, "A method for the construction of minimumredundancy codes," in Proc. of the IRE, 1952, pp. 1098-1101.

[13] N. Eagle, A. Pentland, and D. Lazer, "Inferring social network structure using mobile phone data," PNAS, vol. 106, no. 36, pp. $15274-15278,2009$.

[14] B. Gallagher, H. Tong, T. Eliassi-Rad, and C. Faloutsos, "Using ghost edges for classification in sparsely labeled networks," in $K D D, 2008$, pp. 256-264.

[15] R. N. Lichtenwalter, J. T. Lussier, and N. V. Chawla, "New perspectives and methods in link prediction," in $K D D, 2010$, pp. 243-252.

[16] K. Henderson, T. Eliassi-Rad, C. Faloutsos, L. Akoglu, L. Li, K. Maruhashi, B. A. Prakash, and H. Tong, "Metric forensics: A multi-level approach for mining volatile graphs," in $K D D$, 2010, pp. 163-172.
[17] L. Akoglu, M. McGlohon, and C. Faloutsos, "Oddball: Spotting anomalies in weighted graphs," in $P A K D D, 2010$, pp. $410-421$.

[18] B. Gallagher and T. Eliassi-Rad, "Leveraging labelindependent features for classification in sparsely labeled networks: An empirical study," Lecture Notes in Computer Science, vol. 5498, pp. 1-19, 2010.

[19] D. Cai, C. Zhang, and X. He, "Unsupervised feature selection for multi-cluster data," in $K D D, 2010$, pp. 333-342.

[20] X. Kong and P. S. Yu, "Multi-label feature selection for graph classification," in ICDM, 2010, pp. 274-283.

[21] H. Fei and J. Huan, "Structure feature selection for graph classification," in CIKM, 2008, pp. 991-1000.

[22] A. Mccallum, X. Wang, and A. Corrada-Emmanuel, "Topic and role discovery in social networks with experiments on enron and academic email," JAIR, vol. 30, no. 1, pp. 249$272,2007$.

[23] M. Somaiya, C. Jermaine, and S. Ranka, "Mixture models for learning low-dimensional roles in high-dimensional data," in KDD, 2010, pp. 909-918.

[24] H. Fürstenau and M. Lapata, "Semi-supervised semantic role labeling," in Proc. of the 12th Conf. of the European Chapter of the Association for Computational Linguistics (EACL), 2009, pp. 220-228.

[25] I. Molloy, N. Li, T. Li, Z. Mao, Q. Wang, and J. Lobo, "Evaluating role mining algorithms," in Proc. of the 14th ACM Symp. on Access Control Models and Technologies (SACMAT), 2009, pp. 95-104.

[26] M. Herbster, "Exploiting cluster-structure to predict the labeling of a graph," in Proc. of the 19th Int'l Conf. on Algorithmic Learning Theory (ALT), 2008, pp. 54-69.

[27] J. Weston, C. Leslie, E. Ie, D. Zhou, A. Elisseeff, and W. S. Noble, "Semi-supervised protein classification using cluster kernels," Bioinformatics, vol. 21, pp. 3241-3247, 2005.

[28] B. Cao, N. Liu, and Q. Yang, "Transfer learning for collective link prediction in multiple heterogenous domains," in $I C M L$, 2010, pp. 159-166.

[29] W. Dai, O. Jin, G.-R. Xue, Q. Yang, and Y. Yu, "Eigentransfer: a unified framework for transfer learning," in ICML, 2009, pp. 193-200.

[30] Z. Wang, Y. Song, and C. Zhang, "Knowledge transfer on hybrid graph,” in IJCAI, 2009, pp. 1291-1296.

[31] I. Bhattacharya, S. Godbole, S. Joshi, and A. Verma, "Crossguided clustering: Transfer of relevant supervision across domains for improved clustering," in ICDM, 2009, pp. 4150 . 